04

\title{
Оптические постоянные монокристалла апатита в ИК области 6-28 $\mu \mathrm{m}$
}

\author{
(C) В.М. Золотарев \\ Университет ИТМО, \\ 197101 Санкт-Петербург, Россия \\ e-mail: VM-Zolotarev@mail.ru
}

Поступила в редакцию 25.09.2017 г.

На основе измеренных поляризованных ИК спектров отражения в области $5000-350 \mathrm{~cm}^{-1}$, полученных от естественной грани оптически прозрачного монокристалла фторапатита, методом Крамерса-Кронига вычислены составляющие комплексного показателя преломления (оптические постоянные) для ориентаций вектора излучения $\mathbf{E} \| \mathbf{c}$ и $\mathbf{E} \perp \mathbf{c}$. Монокристалл фторапатита был отобран из нескольких образцов, содержал минимальное количество примесей и обладал высокой степенью кристалличности в соответствии с критериями инфракрасной (ИК) спектроскопии и спектроскопии комбинационного рассеяния. Табличные данные оптических постоянных для обыкновенного и необыкновенного лучей приведены для ИК области 6-28 $\mu \mathrm{m}$. Проведено сравнение полученных спектров абсорбции с результатами квантово-химических расчетов, выполненных методом $a b$ initio в рамках B3LYP-моделирования.

DOI: $10.21883 /$ OS.2018.02.45534.213-17

Физико-химические свойства апатитов, имеющих формулу вида $\mathrm{Ca}_{5}\left(\mathrm{PO}_{4}\right)_{3}(\mathrm{~F}, \mathrm{OH}, \mathrm{Cl})$, активно исследуются с использованием всего арсенала физических методов [14]. Этот интерес к данному минералу обусловлен вариабельностью свойств апатитов, что находит применение в разных отраслях науки и техники. Следует также отметить, что интерес к изучению апатита обусловлен и тем обстоятельством, что он является минералом, входящим в состав костей и зубов всех животных[5-7]. Вариабельность свойств апатитов зависит от содержания в нем конкретных примесей, изоморфно замещающих катионы $\mathrm{Ca}^{2+}$ и анионы $\mathrm{PO}_{4}^{3-}$, которые являются основными элементами, формирующими кристаллическую решетку апатита. Методы инфракрасной (ИК) спектроскопии наиболее часто используются при изучении природных апатитов минерального происхождения [8-12]. Преобладающее большинство исследований, проводимых с помощью ИК спектроскопии, выполнено на качественном уровне [10-16]. Вместе с тем для сопоставления результатов расчетов спектров апатитов с разными заместителями катионов и анионов, выполненных в режиме $a b$ initio, полезно знать для средней ИК области абсолютные значения величин поглощения и преломления апатитов [17,18]. Такая информация имеется для эмали дентина зуба в терагерцовой [19] и для апатита в микроволновой [20] области спектра, для которой отмечается необычный характер зависимости диэлектрической постоянной апатита от частоты по сравнению с другими минералами. Эта особенность касается частотной зависимости диэлектрической постоянной $(\varepsilon)$ и ее высокого значения для образца исследованного апатита, а также сильной анизотропии $\varepsilon$. Так, согласно [20], для $\varepsilon_{\|}=7.4, \varepsilon_{\perp}=9.5$, где индексы $\|$ и $\perp$ означают ориентацию электрического вектора $\mathbf{E}$ внешнего электромагнитного поля по отношению к оси с кристалла, которая совпадает с направлением оптической оси этого кристалла. Для средней ИК области информация по оптическим постоянным апатита практически отсутствует, за исключением работы [21], в которой данные, полученные методом классического дисперсионного анализа, представлены в виде параметров осцилляторов, с помощью которых описывалась спектральная кривая коэффициента отражения $R(v)$. Однако имеются числовые данные, касающиеся измерений на эмали дентина зуба, применительно к длинам волн 9.6, 10.3 и $10.6 \mu \mathrm{m} \mathrm{CO}$-лазера [22,23]. Поскольку эмаль дентина состоит на 98\% из апатита [14], эти данные можно сопоставить с измерениями для апатита. Отсутствие в справочной литературе данных по оптическим постоянным апатита можно объяснить нестабильностью его свойств, зависящих от месторождения минерала и соответственно от примесей, сопутствующих данному месторождению. Вместе с тем сведения по отражению кристаллического апатита достаточно широко представлены в средней ИК областии обычно получены для ориентированных кристаллов фторапатита (FAp) с использованием поляризованного [21] и неполяризованного $[24,25]$ излучений. Эти данные по значению величины коэффициента отражения $R$ сильно отличаются между собой (табл. 1). Соответствующие различия величины $R$ для разных источников $[21,24,25]$ зависят не только от месторождения минерала, что обусловлено рядом факторов: дефектами кристаллической структуры апатита, вкраплениями другой кристаллической фазы, особенностями рельефа и формы отражающей поверхности образца. Дополнительный фактор связан с различиями в качестве полировки образцов и в ориентации кристалла по отношению к электрическому вектору падающего излучения. Влияние перечисленных факторов на результаты измерений $R$ имеют место как для обработанных 
Таблица 1. Характеристики монокристаллов апатита

\begin{tabular}{|c|c|c|c|c|c|c|}
\hline \multirow[b]{2}{*}{ Образцы } & \multirow[b]{2}{*}{ Название } & \multirow[b]{2}{*}{ Измеренный химический состав } & \multicolumn{2}{|c|}{ ИК спектр $R$} & \multirow[b]{2}{*}{ Габитус и цвет } & \multirow[b]{2}{*}{ Происхождение } \\
\hline & & & $\begin{array}{c}R_{\min } \\
1172 \mathrm{~cm}-1\end{array}$ & $\begin{array}{c}R_{\max } \\
1063 \mathrm{~cm}-1\end{array}$ & & \\
\hline S1 & CFAp & $\mathrm{Ca}_{5}\left(\mathrm{PO}_{4}, \mathrm{CO}_{3}\right)_{3} \mathrm{~F}$ & 0.0153 & 0.692 & $\begin{array}{l}\text { Призматическая призма, } \\
\text { темно-серый }\end{array}$ & $\begin{array}{l}\text { Россия, Прибайкалье, } \\
\text { Слюдянка }\end{array}$ \\
\hline S2 & FAp & $\mathrm{Ca}_{5.00}\left(\mathrm{P}_{1.00} \mathrm{O}_{4}\right)_{3}\left(\mathrm{~F}_{0.98} \mathrm{OH}_{0.01} \mathrm{Cl}_{0.01}\right)$ & 0.00035 & $\frac{0.808}{0.755}$ & $\begin{array}{l}\text { Призматическая призма, } \\
\text { двойниковый, прозрачный }\end{array}$ & $\begin{array}{l}\text { Россия, Хибины, } \\
\text { Кольский полуостров }\end{array}$ \\
\hline S3 & FAp & $\mathrm{Ca}_{5.00}\left(\mathrm{P}_{1.00} \mathrm{O}_{4}, \mathrm{CO}_{3}\right)_{3}\left(\mathrm{~F}_{0.90} \mathrm{OH}_{0.1}\right)$ & 0.00034 & 0.755 & $\begin{array}{l}\text { Таблитчатый, } \\
\text { облитерированный, } \\
\text { прозрачный }\end{array}$ & $\begin{array}{l}\text { Россия, Апатиты, } \\
\text { Кольский полуостров }\end{array}$ \\
\hline S4 & FAp & $\begin{array}{l}\left(\mathrm{Ca}_{4.4} \mathrm{Sm}_{0.30} \mathrm{Mn}_{0.10} \mathrm{Na}_{0.15} \mathrm{Cs}_{0.05}\right)_{\Sigma=5} \\
\quad \times\left(\left(\mathrm{P}_{0.96} \mathrm{Si}_{0.04}\right)_{\Sigma=1} \mathrm{O}_{4}\right)_{3} \mathrm{~F}_{1.00}\end{array}$ & 0.0462 & 0.528 & $\begin{array}{l}\text { Призматическая призма, } \\
\text { синезеленый, непрозрачный }\end{array}$ & $\begin{array}{l}\text { Россия, Хибины, } \\
\text { Кольский полуостров }\end{array}$ \\
\hline S5 & FAp & $\mathrm{Ca}_{5.00}\left(\left(\mathrm{P}_{0.98} \mathrm{Si}_{0.01} \mathrm{~S}_{0.01}\right) \mathrm{O}_{4}\right)_{3} \mathrm{~F}_{1.00}$ & -0.0141 & 1.0002 & Призматическая призма & Durango, Mexico [24] \\
\hline S6 & Ap & $\mathrm{Ca}_{5}\left(\mathrm{PO}_{4}\right)_{3}(\mathrm{OH}, \mathrm{F}, \mathrm{Cl})$ & $<0.01$ & $\sim 0.23$ & $\begin{array}{l}\text { Призматическая призма, } \\
\text { непрозрачный }\end{array}$ & $\begin{array}{l}\text { Россия, Прибайкалье, } \\
\text { Слюдянка [25] }\end{array}$ \\
\hline S7 & FAp & $\mathrm{Ca}_{5.00}\left(\left(\mathrm{P}_{0.98} \mathrm{Si}_{0.01} \mathrm{~S}_{0.01}\right) \mathrm{O}_{4}\right)_{3} \mathrm{~F}_{1.00}$ & $\sim \frac{0.02}{0.03}$ & $\sim \frac{0.73}{0.60}$ & $\begin{array}{l}\text { Светло-желтый, короткая } \\
\text { гексагональная призма }\end{array}$ & Durango, Mexico [21] \\
\hline S8 & FAp & $\begin{array}{l}\left(\mathrm{Ca}_{4.68} 0.32\right)_{\Sigma=5} \\
\quad \times\left(\left(\mathrm{PO}_{4}\right)_{2.36}\left(\mathrm{CO}_{3}\right)_{0.64}\right)_{\Sigma=3} \mathrm{~F}_{1.00}\end{array}$ & -...- & $-\ldots$ & $\begin{array}{l}\text { Светло-коричневый, } \\
\text { кистевидный } \\
\text { агрегированный }\end{array}$ & $\begin{array}{l}\text { Limburg de an Lahn, } \\
\text { Germany }[28]\end{array}$ \\
\hline S9 & FAp & $\mathrm{Ca}_{5.00}\left(\mathrm{P}_{1.00} \mathrm{O}_{4}\right) 3\left(\mathrm{~F}_{0.86} \mathrm{Cl}_{0.13}\right)$ & - - - - & - - - - & $\begin{array}{l}\text { Светло-зеленый, } \\
\text { гексагональная призма }\end{array}$ & $\begin{array}{l}\text { Fulford, Eagle County, } \\
\text { Colorado, USA [28] }\end{array}$ \\
\hline
\end{tabular}

Примечание. Значения $R$ образцов $\mathrm{S} 1-\mathrm{S} 4$ даны для ориентации $\mathbf{E} \| \mathbf{c}$. В колонках „ИК спектр $R^{“}$ числитель дроби соответствует значениям $R$ тах для ориентации $\mathbf{E} \| \mathbf{c}$, а знаменатель - для $\mathbf{E} \perp \mathbf{c}$. Значения $R$ образцов $\mathrm{S} 5, \mathrm{~S} 6$ даны для неполяризованного излучения. Значения $R$ образца $\mathrm{S} 5$ получены для грани кристалла (0001), образцы S5-S7 полировались.

поверхностей (полировка), так и для необработанных (естественные грани кристалла). В связи с вышесказанным поиск образца апатита с бездефектной кристаллической решеткой, обладающего совершенной огранкой, которая характеризуется гладкими и плоскими гранями, представляется непростой задачей.

Цель настоящей работы состоит в измерении оптических постоянных монокристалла апатита в средней ИК области методом отражения для образца, обладающего высокой кристалличностью и содержащего минимальное количество примесей.

\section{Образцы и аппаратура}

Исследуемые образцы S1-S4 (табл. 1) представляли кристаллы фторапатита (FAp) и карбонат-фторапатита (CFAp) и имели четко выраженную гексагональную огранку. Ориентировка образцов проводилась под микроскопом относительно ребер кристалла. Отражение измерялось от естественной необработанной грани [0101]. Излучение направлялось вблизи нормали относительно грани образца [0101].

Спектры комбинационного рассеяния (КР) в диапазоне $1400-45 \mathrm{~cm}^{-1}$ с разрешением $3 \mathrm{~cm}^{-1}$ измерялись с помощью дисперсионного КР-микроскопа SENTERRA фирмы Bruker, снабженного конфокальным оптическим микроскопом Olympus BX-51 и объективами с увеличением $50 \times$ и $100 \times$. Возбуждение спектра КР осуществлялось на длине волны $532 \mathrm{~nm}$ с помощью неодимового лазера (вторая гармоника YAG: Nd-лазера).

Спектры ИК отражения образцов апатитов в диапазоне 5000-350 $\mathrm{cm}^{-1}$ были получены на ИК Фурьеспектрометре TENSOR 27 фирмы Bruker с разрешением $2 \mathrm{~cm}^{-1}$ для диафрагмы $2 \mathrm{~mm}$ при 500 сканах в поляризованном излучении. Использовались дифракционные поляризаторы 1200 и 600 lines/mm на подложках: стекло ИКС-35 и пленка из полиэтилена - PE.

Для оценки качества исследованных кристаллов апатита использовались методы КР и ИК спектроскопии отражения. В спектре КР спектроскопические параметры полосы $965 \mathrm{~cm}^{-1}$ симметричных колебаний $v_{1}$ чувствительны к дефектности [26] кристаллической решетки апатита и степени ее кристалличности [27]. Увеличение дефектности кристалла и уменьшение кристалличности приводит к увеличению в 2-3 раза полуширины $\Delta v_{1}$ полосы $v_{1}$ и смещению ее в низкочастотную сторону на величину до $\sim 5 \mathrm{~cm}^{-1}$ по мере уменьшения величины кристалличности. На рис. 1 приведены спектры КР поло- 
сы $v_{1}$ исследованных образцов S1, S2, S4 (вставка вверху справа) и в центральной части рис. 1 спектры 2-4 апатитов из базы данных RRUFF [28] в сопоставлении со спектром 1 (образец S2, табл. 1) Из приведенных данных видно, что полоса $v_{1}$ в спектре КР у образца S2 наиболее узкая из всех сопоставляемых образцов и имеет наиболее высокое значение частоты $v_{1}=965 \mathrm{~cm}^{-1}$. Полосы деформационных колебаний $v_{2}$ и $v_{4}$ и фононный спектр апатита еще более чувствительны к деформации тетраэдра $\mathrm{PO}_{4}$ и кристаллической решетки в целом, что наглядно проявляется в диапазонах 650-400 и 400-45 $\mathrm{cm}^{-1}$ соответственно (рис. 2). Следует отметить, что частоты $v_{1}, v_{2}, v_{3}$ и $v_{4}$ в поляризационных

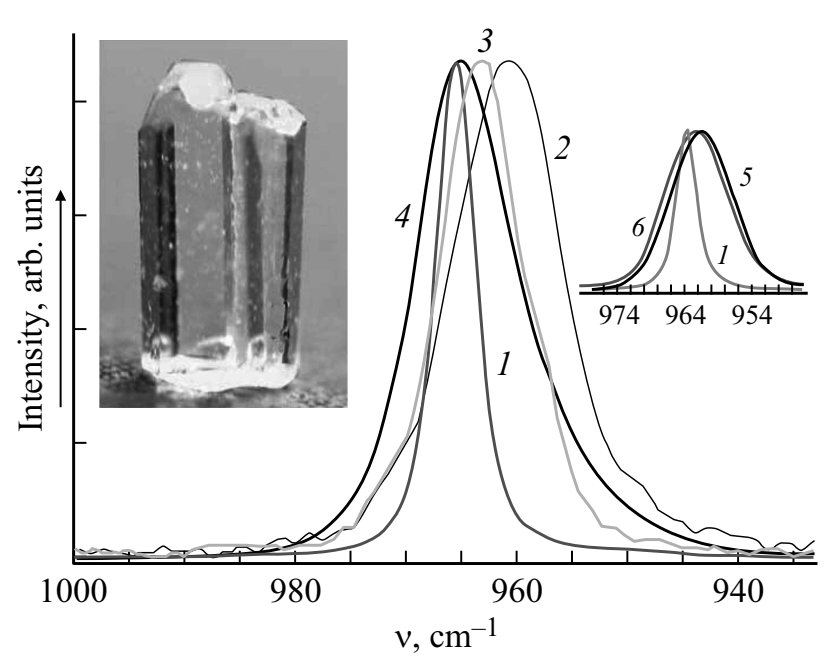

Рис. 1. Спектры КР монокристаллов апатита в области полосы $965 \mathrm{~cm}^{-1}$ симметричных колебаний $\nu_{1}$ аниона $\mathrm{PO}_{4}^{3-}$. Спектр 1 - образец S2 (табл. 1). Спектры $2-4-$ из базы данных RRUFF [28], 2 - R050274, 3 - R050617, 4 $\mathrm{R} 050529$. В верхней части рисунка слева показано фото образца S2. Вверху справа показана полоса $v_{1}$ трех исследованных образцов. Спектры 1,5,6 соответствуют образцам S2-S1-S4 соответственно (табл. 1).

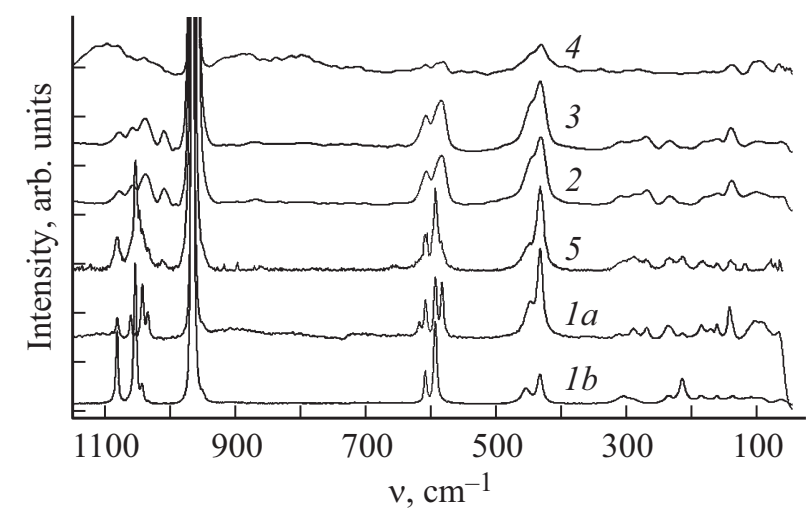

Рис. 2. Спектры КР монокристаллов апатита в области $1200-45 \mathrm{~cm}^{-1}$. Спектры $1-4$ получены для ориентаций $(1 a-4)-\mathbf{E} \perp \mathbf{c}, 1 b-\mathbf{E} \| \mathbf{c}, 5-$ спектр для неполяризованного излучения. Спектры $1-5$ соответствуют образцам S2, S3, $\mathrm{S} 1, \mathrm{~S} 4$ и S5 соответственно (табл. 1).

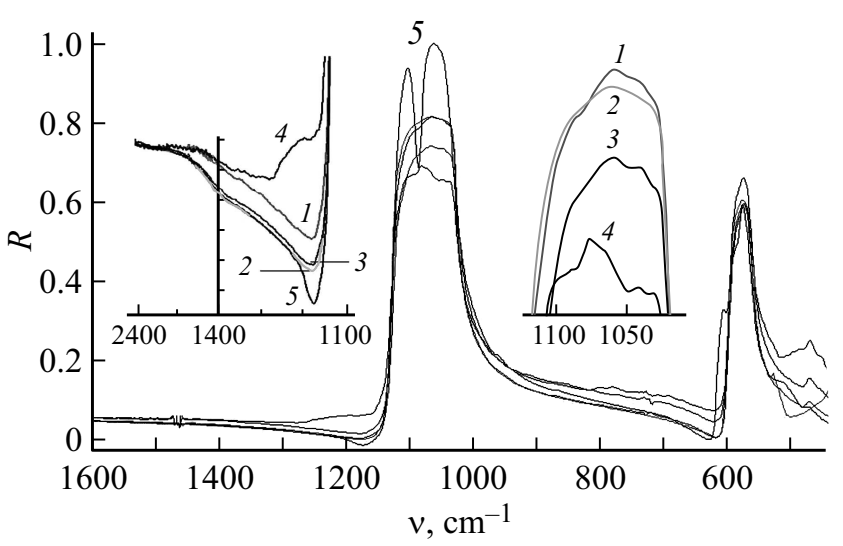

Рис. 3. Спектры ИК отражения R монокристаллов апатита в области полосы $1095 \mathrm{~cm}^{-1}$ антисимметричных колебаний $v_{3}$ аниона $\mathrm{PO}_{4}^{3-}$. Нумерация спектров соответствует нумерации образцов в табл. $1: 1-\mathrm{S} 1,2-\mathrm{S} 2,3-\mathrm{S} 3,4-\mathrm{S} 4,5-$ S5. Спектры 1-4 получены отражением от грани [0101] для вектора $\mathbf{E} \| \mathbf{c}$, спектр 5 - излучение неполяризовано. В левой части показан с растяжкой по шкале ординат участок спектра в окрестности минимума $R$. В правой части показан с растяжкой по шкале ординат участок спектра в окрестности максимума $R$.

спектрах КР образцов S2 и S7 (аналог S5) (табл. 1) для ориентаций электрического вектора возбуждающего излучения $\mathbf{E}_{\|}$и $\mathbf{E}_{\perp}$ полностью совпали по частоте (погрешность в среднем не превышала $1 \mathrm{~cm}^{-1}$ ). Данные для частот $v_{1}, v_{2}, v_{3}$ и $v_{4}$ образца S7 (месторождение Durango, Mexico) приведены в [21]. С учетом малой ширины полос в спектре КР сравниваемых образцов это указывает на совершенство кристаллической решетки обеих образцов S2 и S5, что проявляется также и в сходстве их фононных спектров (рис. 2, спектры 1 и 5 ). В спектре ИК абсорбции параметры дублета 1029 и $\sim 1095 \mathrm{~cm}^{-1}$, относящегося к антисимметричным колебаниям $v_{3}$, подобным колебанию $v_{1}$ в спектре КР, реагируют на дефектность кристаллической решетки апатита и ее кристалличность [18]. Рост дефектности кристалла и уменьшение кристалличности приводит к уширению полосы $v_{3}$ и уменьшению ее интенсивности. Эти особенности наиболее заметно проявляются в окрестности минимума отражения, где на порядки меняется величина коэффициента отражения $R$ (рис. 3, табл. 1). Увеличение полуширины $\Delta v_{3}$ полосы $1029 \mathrm{~cm}^{-1}$ уменьшает крутизну ее высокочастотного крыла, что приводит к снижению контраста коэффициента $R\left(\Delta R=R_{\max }-R_{\min }\right)$ в окрестности полосы $v_{3}$. Значения параметра $R_{\min }$ сильно влияют на величины $n$ и $k$ комплексного показателя преломления $(\hat{n}=n-i k)$ в окрестности $R_{\min }$ при их определении методом Крамерса-Кронига, поскольку в интегральном выражении величина $R$ стоит под знаком логарифма.

Совокупность данных КР и ИК спектроскопии для образцов S1-S4 и S5, S7, а также их характеристики (табл. 1) позволяют с учетом данных [21] сделать заключение о высоком качестве кристаллической решетки образца S2, который на этом основании был выбран 
в качестве основного объекта для работ, связанных с определением оптических постоянных $n$ и $k$ монокристалла FAp.

\section{Методика исследований}

Большое значение величины коэффициента отражения $R$ апатитов в средней ИК области $4000-400 \mathrm{~cm}^{-1}$ благоприятствует применению метод Крамерса-Кронига (КК) для определения оптических постоянных $n$ и $k$. Однако вблизи края низкочастотного диапазона, т.е. в длинноволновой ИК области 400-2 $\mathrm{cm}^{-1}$, имеется очень сильное поглощение кристаллической решетки апатита. Учет области 400-2 $\mathrm{cm}^{-1}$ необходим для корректных вычислений значений $n$ и $k$ из спектра $R$ методом КК $[29,30]$ в диапазоне $4000-400 \mathrm{~cm}^{-1}$. Значения $R$ для области 400-2 $\mathrm{cm}^{-1}$ находились путем предварительного расчета $n$ на основе значений $k$ для этого диапазона частот с помощью уравнения

$$
n\left(v_{0}\right)=C\left(v_{0}\right)+\frac{2}{\pi} \int_{v_{\mathrm{H}}}^{v_{\mathrm{K}}} \frac{k(v) v d v}{v^{2}-v_{0}^{2}},
$$

$n\left(v_{0}\right)$ - показатель преломления для фиксированной частоты $v_{0} ; v$ - текущая частота внутри измеряемого спектрального интервала $v_{\text {н }}-v_{\text {K }}$; $v_{\text {н }}$ и $v_{\mathrm{K}}-$ начальная и конечная частоты измеряемого спектрального интервала; $C\left(v_{0}\right)$ - поправка, учитывающая конечность интервала интегрирования.

Необходимые для расчета $n$ величины $k$ находились путем нормировки величин абсорбции (оптической плотности), полученных в работе [17], к абсолютным значениям $k$ в области $400-2 \mathrm{~cm}^{-1}$, что осуществлялось путем привязки основного максимума $293 \mathrm{~cm}^{-1}$ в спектре абсорбции, полученного в работе методом $a b$ initio для гидроксилапатита в области $1300-0 \mathrm{~cm}^{-1}$ [17], к полосе $601 \mathrm{~cm}^{-1}$, для которой была измерена величина $k$ в максимуме. Значения $k$ для полосы $601 \mathrm{~cm}^{-1}$ были предварительно найдены в первом приближении без учета диапазона $400-2 \mathrm{~cm}^{-1}$ из экспериментального спектра $R$ для области $5000-400 \mathrm{~cm}^{-1}$ с помощью уравнения

$$
\varphi\left(v_{0}\right)=-\frac{v_{0}}{\pi} \int_{v_{\mathrm{H}}}^{v_{\mathrm{K}}} \frac{\ln R(v) d v}{v^{2}-v_{0}^{2}}+C^{\prime}\left(v_{0}\right)+C^{\prime \prime}\left(v_{0}\right),
$$

$\varphi\left(v_{0}\right)$ - фазовый сдвиг отраженной волны для фиксированной частоты $v_{0} ; R=|\hat{r}|^{2}$ - измеряемый коэффициент отражения; $\hat{r}\left(v_{0}\right)$ - амплитудный коэффициент отражения; $C^{\prime}\left(v_{0}\right), C^{\prime \prime}\left(v_{0}\right)$ - поправки, учитывающие конечность интервала интегрирования.

Первая поправка учитывает вклад от участков спектра, находящихся за пределами интервала измерений

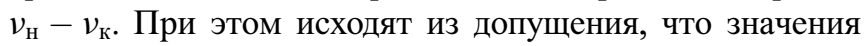
$R(v)$ в этих участках постоянны и равны значениям

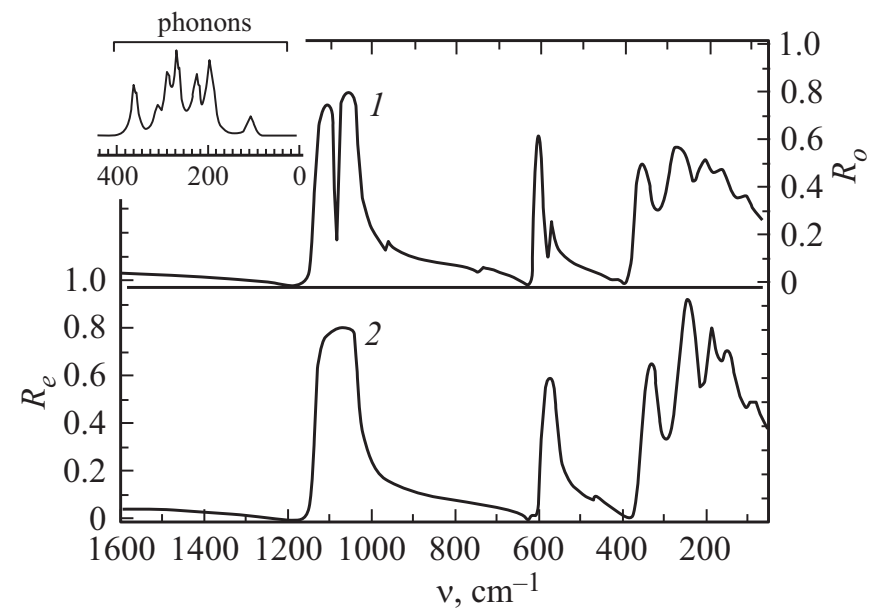

Рис. 4. Спектры ИК отражения $R$ монокристалла фторапатита (образец S2): спектр 1 получен для вектора $\mathbf{E} \perp \mathbf{c}$ $\left(R_{o}\right.$ - обыкновенный луч), спектр 2 - для вектора $\mathbf{E} \| \mathbf{c}$ $\left(R_{e}-\right.$ необыкновенный луч). В левой части вверху показан участок спектра поглощения апатита в длинноволновой области спектра, полученный теоретическим расчетом [17] в рамках метода $a b$ initio.

$R\left(v_{\mathrm{H}}\right)$ и $R\left(v_{\mathrm{\kappa}}\right)$. Отступление от этого допущения учитывается вторым поправочным слагаемым $C^{\prime \prime}\left(v_{0}\right)$, которое находится на основе независимых измерений оптических постоянных $n$ и $k$ в двух, „привязочных“ точках $v_{0}^{\prime}$ и $v_{0}^{\prime \prime}$ слева и справа от интервала $v_{\text {н }}-v_{\text {к }}$.

После определения $n$ по уравнению (1) значения $R$ для диапазона $400-2 \mathrm{~cm}^{-1}$ находились расчетом по формуле Френеля для нормального падения на основе полученных оптических постоянных $n$ и $k$ :

$$
R=|r|^{2}=\frac{(n-1)^{2}+\kappa^{2}}{(n+1)^{2}+\kappa^{2}}
$$

где $r$ - амплитудный коэффициент отражения, определяемый из уравнения (3):

$$
r=\frac{1-n-i k}{1+n+i k}=|r| e^{i \varphi} .
$$

Полученные с помощью уравнения (3) значения $R$ для диапазона 400-2 $\mathrm{cm}^{-1}$ сшивались с экспериментальными величинами $R_{e}$ и $R_{o}$, измеренными в диапазоне $5000-350 \mathrm{~cm}^{-1}$ соответственно для двух ориентаций $(\|, \perp)$ вектора $\mathbf{E}$ относительно оси с кристалла (рис. 4). После этого с помощью уравнения (2) проводились по стандартной программе OPUS 7.5 вычисления фазового сдвига $\varphi(v)$ последовательно для обеих величин $R_{e}$ и $R_{o}$ в диапазоне $5000-2 \mathrm{~cm}^{-1}$. В качестве величины $n_{0}^{\prime}$ использовалось значение $n_{D}$ для области прозрачности $\operatorname{FAp}\left(n_{D \|}=1.633, n_{D \perp}=1.630\right.$, индексы $\|$ и $\perp$ относятся к необыкновенному и обыкновенному лучам соответственно), $k_{0}^{\prime}=0$. Для низкочастотного интервала принималось значение $n_{0}^{\prime \prime}=\sqrt{\varepsilon}[20]$, где $\varepsilon-$ диэлектрическая проницаемость $\left(\varepsilon_{\|}=7.4, \varepsilon_{\perp}=9.5\right), k_{0}^{\prime \prime} \approx 0$. Вычислив с 
Таблица 2. Оптические постоянные фторапатита $(\mathbf{E} \perp \mathbf{c}$, обыкновенный луч)

\begin{tabular}{|c|c|c|c|c|c|c|c|c|}
\hline$v, \mathrm{~cm}^{-1}$ & $n_{o}$ & $k_{o}$ & $v, \mathrm{~cm}^{-1}$ & $n_{o}$ & $k_{o}$ & $v, \mathrm{~cm}^{-1}$ & $n_{o}$ & $k_{o}$ \\
\hline 350 & 0.938 & 1.747 & 720 & 1.630 & 0.096 & 1076 & 0.335 & 1.610 \\
\hline 360 & 0.575 & 1.523 & 740 & 1.677 & 0.169 & 1078 & 0.347 & 1.458 \\
\hline 380 & 0.398 & 0.797 & 750 & 1.579 & 0.162 & 1080 & 0.399 & 1.281 \\
\hline 400 & 0.781 & 0.229 & 760 & 1.622 & 0.078 & 1082 & 0.525 & 1.107 \\
\hline 420 & 1.175 & 0.164 & 780 & 1.702 & 0.067 & 1084 & 0.749 & 0.971 \\
\hline 440 & 1.296 & 0.176 & 800 & 1.744 & 0.070 & 1086 & 1.080 & 0.972 \\
\hline 460 & 1.458 & 0.176 & 820 & 1.784 & 0.069 & 1088 & 1.413 & 1.250 \\
\hline 480 & 1.565 & 0.164 & 840 & 1.828 & 0.075 & 1090 & 1.397 & 1.761 \\
\hline 500 & 1.654 & 0.177 & 860 & 1.865 & 0.074 & 1092 & 1.011 & 2.039 \\
\hline 540 & 1.915 & 0.220 & 900 & 1.971 & 0.072 & 1094 & 0.658 & 1.978 \\
\hline 550 & 2.008 & 0.275 & 940 & 2.147 & 0.080 & 1096 & 0.472 & 1.834 \\
\hline 560 & 2.074 & 0.374 & 950 & 2.216 & 0.086 & 1098 & 0.374 & 1.693 \\
\hline 570 & 2.385 & 0.571 & 960 & 2.348 & 0.137 & 1100 & 0.320 & 1.580 \\
\hline 572 & 2.453 & 0.739 & 970 & 2.193 & 0.145 & 1110 & 0.207 & 1.173 \\
\hline 574 & 2.432 & 0.997 & 980 & 2.386 & 0.089 & 1120 & 0.176 & 0.857 \\
\hline 576 & 2.185 & 1.233 & 990 & 2.544 & 0.102 & 1130 & 0.192 & 0.545 \\
\hline 578 & 1.844 & 1.221 & 1000 & 2.736 & 0.122 & 1140 & 0.354 & 0.214 \\
\hline 580 & 1.631 & 1.008 & 1010 & 3.031 & 0.156 & 1150 & 0.611 & 0.100 \\
\hline 582 & 1.612 & 0.761 & 1020 & 3.557 & 0.260 & 1160 & 0.767 & 0.074 \\
\hline 584 & 1.720 & 0.581 & 1022 & 3.710 & 0.300 & 1180 & 0.953 & 0.058 \\
\hline 586 & 1.894 & 0.493 & 1024 & 3.893 & 0.352 & 1200 & 1.067 & 0.047 \\
\hline 588 & 2.081 & 0.489 & 1026 & 4.126 & 0.415 & 1220 & 1.152 & 0.047 \\
\hline 590 & 2.281 & 0.542 & 1028 & 4.426 & 0.529 & 1260 & 1.264 & 0.040 \\
\hline 592 & 2.504 & 0.676 & 1030 & 4.815 & 0.717 & 1300 & 1.333 & 0.037 \\
\hline 594 & 2.752 & 0.939 & 1032 & 5.320 & 1.073 & 1340 & 1.382 & 0.034 \\
\hline 596 & 2.930 & 2.211 & 1034 & 5.931 & 1.803 & 1380 & 1.421 & 0.034 \\
\hline 600 & 1.920 & 2.615 & 1036 & 6.197 & 3.229 & 1420 & 1.451 & 0.036 \\
\hline 602 & 1.222 & 2.464 & 1038 & 5.317 & 4.778 & 1460 & 1.471 & 0.034 \\
\hline 604 & 0.825 & 2.137 & 1040 & 3.779 & 5.284 & 1500 & 1.488 & 0.034 \\
\hline 606 & 0.615 & 1.834 & 1042 & 2.662 & 5.024 & 1540 & 1.502 & 0.030 \\
\hline 608 & 0.492 & 1.571 & 1044 & 1.993 & 4.602 & 1580 & 1.523 & 0.017 \\
\hline 610 & 0.418 & 1.341 & 1046 & 1.584 & 4.226 & 1620 & 1.551 & 0.037 \\
\hline 612 & 0.374 & 1.130 & 1048 & 1.300 & 3.898 & 1660 & 1.552 & 0.032 \\
\hline 614 & 0.355 & 0.931 & 1050 & 1.099 & 3.622 & 1700 & 1.553 & 0.044 \\
\hline 616 & 0.364 & 0.738 & 1052 & 0.945 & 3.376 & 1740 & 1.562 & 0.040 \\
\hline 618 & 0.406 & 0.557 & 1054 & 0.829 & 3.166 & 1750 & 1.564 & 0.039 \\
\hline 620 & 0.482 & 0.395 & 1056 & 0.733 & 2.973 & & & \\
\hline 630 & 0.969 & 0.104 & 1058 & 0.659 & 2.805 & & & \\
\hline 640 & 1.192 & 0.087 & 1060 & 0.595 & 2.647 & & & \\
\hline 650 & 1.307 & 0.092 & 1070 & 0.387 & 2.000 & & & \\
\hline 660 & 1.384 & 0.089 & 1072 & 0.362 & 1.874 & & & \\
\hline 700 & 1.567 & 0.078 & 1074 & 0.344 & 1.750 & & & \\
\hline
\end{tabular}

помощью (2) фазовый сдвиг $\varphi\left(v_{0}\right)$ и определив таким образом величину $\hat{r}\left(v_{0}\right)$ можно из формулы Френеля, уравнение (4), определить комплексный показатель преломления и, следовательно, $n$ и $k$ объекта:

$$
\begin{aligned}
& n=\frac{1-R}{1+R-2 \sqrt{R} \cos \varphi}, \\
& k=\frac{-2 \sqrt{R} \sin \varphi}{1+R-2 \sqrt{R} \cos \varphi} .
\end{aligned}
$$

На основе полученных $n$ и $k$ можно вычислить оптическую плотность $(D)$ из соотношения $D=-\lg T=K d$, где $T$ - пропускание, $K-$ коэффициент поглощения,
$K=4 \pi k v\left[\mathrm{~cm}^{-1}\right], d-$ толщина образца $[\mathrm{cm}], v-$ волновое число $\left[\mathrm{cm}^{-1}\right]$.

Результаты расчетов оптических постоянных $n$ и $k$ монокристалла FAp (образец S2) для двух ориентаций вектора $\mathbf{E}$ относительно оси кристалла с представлены на рис. 5, 6 и даны в табл. 2, 3 для ориентаций $\mathbf{E} \perp \mathbf{c}$ (обыкновенный луч) и $\mathbf{E} \| \mathbf{c}$ (необыкновенный луч).

\section{Обсуждение результатов}

Сравнение полученных значений $k$ в области полосы $1032 \mathrm{~cm}^{-1}$ антисимметричных колебаний $v_{3}$ для 
Таблица 3. Оптические постоянные фторапатита (E $\| \mathbf{c}$, необыкновенный луч)

\begin{tabular}{|c|c|c|c|c|c|c|c|c|}
\hline$v, \mathrm{~cm}^{-1}$ & $n_{e}$ & $k_{e}$ & $v, \mathrm{~cm}^{-1}$ & $n_{e}$ & $k_{e}$ & $v, \mathrm{~cm}^{-1}$ & $n_{e}$ & $k_{e}$ \\
\hline 350 & 0.469 & 0.936 & 524 & 2.173 & 0.248 & 958 & 2.324 & 0.0459 \\
\hline 360 & 0.590 & 0.540 & 526 & 2.205 & 0.253 & 960 & 2.342 & 0.047 \\
\hline 380 & 0.992 & 0.252 & 528 & 2.240 & 0.260 & 962 & 2.361 & 0.049 \\
\hline 400 & 1.280 & 0.136 & 530 & 2.279 & 0.271 & 964 & 2.3781 & 0.051 \\
\hline 420 & 1.469 & 0.125 & 532 & 2.323 & 0.287 & 966 & 2.395 & 0.050 \\
\hline 440 & 1.639 & 0.131 & 534 & 2.370 & 0.311 & 968 & 2.416 & 0.048 \\
\hline 442 & 1.650 & 0.133 & 536 & 2.416 & 0.341 & 970 & 2.439 & 0.046 \\
\hline 444 & 1.661 & 0.133 & 538 & 2.463 & 0.376 & 972 & 2.463 & 0.045 \\
\hline 446 & 1.675 & 0.131 & 540 & 2.511 & 0.431 & 974 & 2.489 & 0.043 \\
\hline 448 & 1.692 & 0.130 & 542 & 2.513 & 0.485 & 976 & 2.517 & 0.042 \\
\hline 450 & 1.708 & 0.132 & 544 & 2.533 & 0.459 & 978 & 2.547 & 0.042 \\
\hline 452 & 1.723 & 0.134 & 546 & 2.643 & 0.459 & 980 & 2.579 & 0.042 \\
\hline 454 & 1.740 & 0.135 & 548 & 2.760 & 0.512 & 1000 & 3.053 & 0.066 \\
\hline 456 & 1.759 & 0.138 & 550 & 2.897 & 0.591 & 1020 & 4.699 & 0.322 \\
\hline 458 & 1.779 & 0.143 & 552 & 3.0436 & 0.7243 & 1028 & 6.986 & 3.430 \\
\hline 460 & 1.801 & 0.150 & 554 & 3.199 & 0.917 & 1030 & 5.910 & 5.228 \\
\hline 462 & 1.825 & 0.164 & 556 & 3.337 & 1.211 & 1032 & 4.140 & 5.769 \\
\hline 464 & 1.846 & 0.186 & 558 & 3.395 & 1.616 & 1034 & 2.897 & 5.439 \\
\hline 466 & 1.858 & 0.216 & 560 & 3.273 & 2.107 & 1040 & 1.451 & 4.209 \\
\hline 468 & 1.858 & 0.248 & 566 & 1.924 & 2.795 & 1050 & 0.755 & 3.107 \\
\hline 470 & 1.844 & 0.276 & 570 & 1.225 & 2.501 & 1060 & 0.481 & 2.462 \\
\hline 472 & 1.820 & 0.291 & 580 & 0.583 & 1.659 & 1070 & 0.337 & 1.648 \\
\hline 474 & 1.793 & 0.289 & 590 & 0.381 & 1.001 & 1090 & 0.204 & 1.354 \\
\hline 476 & 1.773 & 0.274 & 600 & 0.529 & 0.374 & 1100 & 0.168 & 1.085 \\
\hline 478 & 1.766 & 0.249 & 610 & 0.901 & 0.146 & 1110 & 0.147 & 0.820 \\
\hline 480 & 1.773 & 0.223 & 620 & 1.099 & 0.110 & 1120 & 0.149 & 0.524 \\
\hline 482 & 1.792 & 0.205 & 640 & 1.310 & 0.088 & 1130 & 0.326 & 0.162 \\
\hline 484 & 1.814 & 0.198 & 660 & 1.422 & 0.075 & 1140 & 0.615 & 0.072 \\
\hline 486 & 1.831 & 0.198 & 700 & 1.559 & 0.055 & 1160 & 0.877 & 0.058 \\
\hline 488 & 1.843 & 0.197 & 760 & 1.706 & 0.047 & 1180 & 1.018 & 0.051 \\
\hline 490 & 1.856 & 0.193 & 800 & 1.785 & 0.045 & 1200 & 1.110 & 0.046 \\
\hline 492 & 1.874 & 0.191 & 860 & 1.910 & 0.055 & 1220 & 1.181 & 0.047 \\
\hline 494 & 1.889 & 0.194 & 900 & 2.017 & 0.056 & 1240 & 1.234 & 0.046 \\
\hline 496 & 1.900 & 0.195 & 930 & 2.134 & 0.051 & 1280 & 1.308 & 0.039 \\
\hline 498 & 1.912 & 0.191 & 932 & 2.143 & 0.051 & 1320 & 1.363 & 0.034 \\
\hline 500 & 1.930 & 0.187 & 934 & 2.153 & 0.049 & 1360 & 1.404 & 0.030 \\
\hline 502 & 1.950 & 0.187 & 936 & 2.164 & 0.047 & 1400 & 1.436 & 0.029 \\
\hline 504 & 1.970 & 0.189 & 938 & 2.176 & 0.046 & 1440 & 1.462 & 0.029 \\
\hline 506 & 1.989 & 0.191 & 940 & 2.189 & 0.045 & 1480 & 1.478 & 0.030 \\
\hline 508 & 2.010 & 0.193 & 942 & 2.203 & 0.044 & 1520 & 1.491 & 0.025 \\
\hline 510 & 2.035 & 0.196 & 944 & 2.218 & 0.044 & 1560 & 1.507 & 0.020 \\
\hline 512 & 2.061 & 0.206 & 946 & 2.233 & 0.045 & 1600 & 1.526 & 0.020 \\
\hline 514 & 2.082 & 0.222 & 948 & 2.247 & 0.0475 & 1640 & 1.536 & 0.021 \\
\hline 516 & 2.096 & 0.235 & 950 & 2.259 & 0.049 & 1680 & 1.550 & 0.016 \\
\hline 518 & 2.109 & 0.242 & 952 & 2.273 & 0.048 & 1720 & 1.559 & 0.022 \\
\hline 520 & 2.126 & 0.245 & 954 & 2.288 & 0.046 & 1750 & 1.565 & 0.023 \\
\hline 522 & 2.147 & 0.247 & 956 & 2.306 & 0.0457 & & & \\
\hline
\end{tabular}

кристалла FАр показало хорошее совпадение (рис. 5) с данными, полученными для эмали дентина методом импульсной фототермальной радиометрии [22]. Численные значения $k$, полученные в [23] для эмали дентина методом Френеля с помощью измерений $R$ при двух углах для ряда длин волн $\mathrm{CO}_{2}$-лазера, показали близкие значения для высокочастотного края полосы $v_{3}$, но заметно отличались в окрестности низкочастотного крыла полосы $v_{3}$. Величина $k$, полученная в [23] для эмали дентина методом экстраполяции в предположении контура Лорентца для полосы $v_{3}$, в максимуме дала значение $k \approx 3.6$, тогда как наш эксперимент для кристалла FAp показал значения $k=5.284-5.769$ для ориентаций $\mathbf{E} \perp \mathbf{c}$ и $\mathbf{E} \| \mathbf{c}$ соответственно. Вместе с тем следует отметить, что для наших измерений $R$, а также в работах $[10,21]$ для максимума $v_{3}$ всех исследованных 
кристаллов FАр выполняется неравенство $R_{e}>R_{o}$. Из этого вытекает необходимость выполнения соотношения $k_{e}>k_{o}$, что согласуется с нашим экспериментом, для которого $k_{e}=5.769$ и $k_{o}=5.284$. Можно отметить, что полученные значения $k_{e}$ и $k_{o}$ для полосы $v_{3}$ FАр близки к соответствующим значениям величин для полосы $v_{3}$ кристаллического кварца $[31,32]$. Это сходство касается и формы сравниваемых полос $v_{3}$ для величин $k_{e}$ и $k_{o}$ в случае FAр и $\alpha-\mathrm{SiO}_{2}$ (рис. 7, вставка вверху слева), что указывает на подобие ориентации тетраэдров $\mathrm{PO}_{4}$ и $\mathrm{SiO}_{4 / 2}$ относительно оптической оси в соответствующих кристаллах. Поскольку частоты основных максимумов

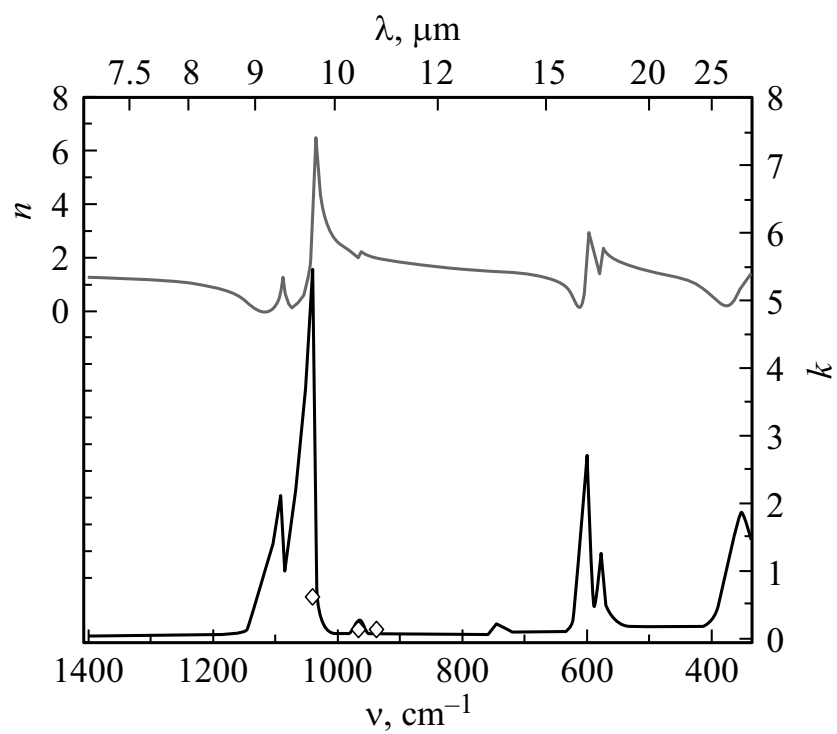

Рис. 5. Оптические постоянные $(n, k)$ монокристалла фторапатита (образец S2) для вектора $\mathbf{E} \perp \mathbf{c}$ (обыкновенный луч). Три точки на спектре $k$ в виде ромбов получены для эмали дентина [22].

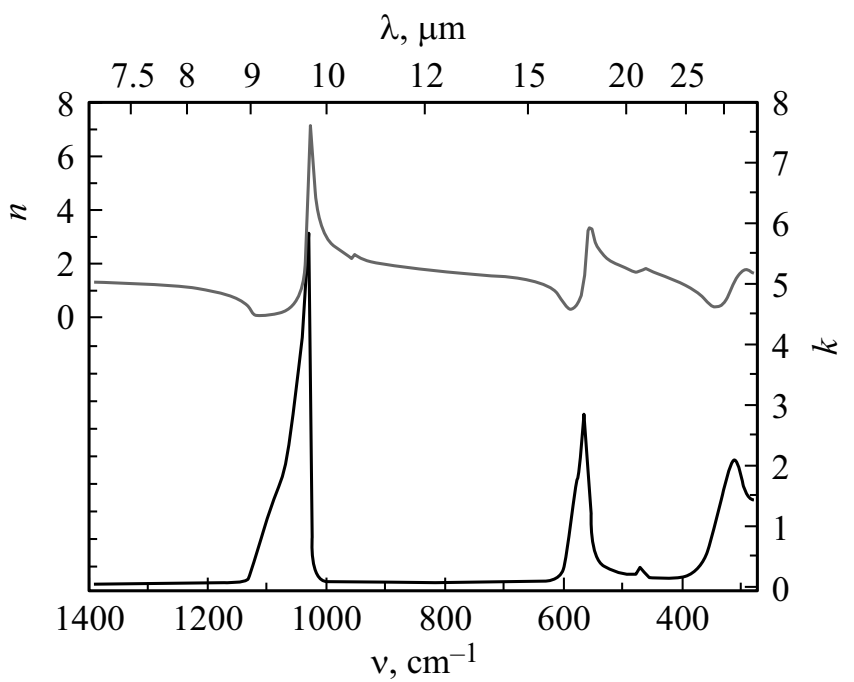

Рис. 6. Оптические постоянные $(n, k)$ монокристалла фторапатита (образец S2) для вектора $\mathbf{E} \| \mathbf{c}$ (необыкновенный луч).

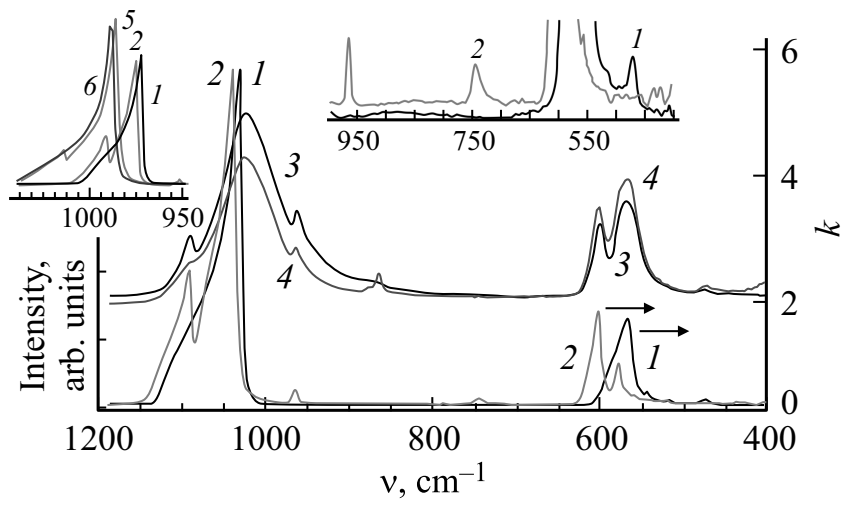

Рис. 7. Сравнение ИК спектров фторапатита (образец S2), полученных методом КК (спектры 1,2, шкала показателя поглощения $k$ справа) и с помощью техники $\mathrm{KBr}$ [28] (спектры 3,4, шкала абсорбции (arb. units)). Спектр 1 получен для вектора $\mathbf{E} \| \mathbf{c}\left(k_{e}-\right.$ необыкновенный луч), спектр $2-$ для вектора $\mathbf{E} \perp \mathbf{c}\left(k_{o}-\right.$ обыкновенный луч). Спектры 3,4 соответствуют образцам S7, S8 (табл. 1) и смещены по оси ординат для удобства сравнения. В центре представлен участок 950-400 $\mathrm{cm}^{-1}$ (спектры 1,2$)$ с $10 \times$ растяжкой по ординате. Слева вверху представлен участок $1250-900 \mathrm{~cm}^{-1}$ (спектры $5-k_{e}, 6-k_{o}$ относятся к кварцу $\alpha-\mathrm{SiO}_{2}$ [32]).

$k_{e}(v)$ и $k_{o}(v)$ валентной антисимметричной полосы $v_{3}$ в отличие от деформационной полосы $v_{2}$ не сильно отличаются для $\mathrm{PO}_{4}$ и $\mathrm{SiO}_{4 / 2}$, то с учетом одинаковой массы колеблющихся атомов О это свидетельствует о близости величины сил валентных связей $\mathrm{P}-\mathrm{O}$ и $\mathrm{Si}-\mathrm{O}$, образующих эти тетраэдры.

Полученные методом КК спектры для показателей поглощения $k_{o}$ и $k_{e}$ полезно сравнить с экспериментальными спектрами абсорбции, которые для образцов апатита в виде порошков обычно получают с помощью техники $\mathrm{KBr}[8,11,14,28]$. Этот способ измерений ИК спектров порошков вносит определенные нелинейные искажения в форму контура наиболее интенсивных полос [33]. Для сравнения на рис. 7 приведены спектры абсорбции (оптическая плотность) FАр, полученные на основе измеренных $k_{o}, k_{e}$ в сопоставлении со спектрами, полученными для FAp в виде порошков с помощью техники $\mathrm{KBr}$ [28]. Спектры образцов S7, S9 (табл. 1) почти не отличаются между собой (спектр 9 на рис. 7 не приводится), тогда как в спектре образца S8 резко уменьшилась интенсивность полосы $v_{3} 1093 \mathrm{~cm}^{-1}$ антисимметричных колебаний тетраэдра $\mathrm{PO}_{4}$ и увеличилась относительно полосы $v_{3}$ интенсивность деформационной полосы $v_{2} 566-601 \mathrm{~cm}^{-1}$. Эти спектроскопические особенности обусловлены сильным понижением симметрии иона $\mathrm{PO}_{4}$ у образца $\mathrm{S} 8$ вследствие внедрения в решетку кристалла FAp иона $\mathrm{CO}_{3}$ путем изоморфного замещения иона $\mathrm{PO}_{4}$ ионом $\mathrm{CO}_{3}$ (табл. 1). Это проявляется в появлении полос 1454-1424 и $866 \mathrm{~cm}^{-1}$, отвечающих колебаниям $v_{1}$ и $v_{2}$ иона $\mathrm{CO}_{3}$ [17] соответственно. Сходство спектров образцов S7 и S9, 
Таблица 4. Частоты полос группы $\mathrm{PO}_{4}^{3-}$ апатита, вычисленные ab initio в гармоническом приближении путем В3LYР-моделирования и определенные экспериментально

\begin{tabular}{|c|c|c|c|c|c|c|c|c|}
\hline \multirow{3}{*}{$\begin{array}{c}\text { Колебательные } \\
\text { моды }\end{array}$} & \multirow{3}{*}{ Активность } & \multirow{3}{*}{ Симметрия } & \multicolumn{3}{|c|}{ Расчет } & \multicolumn{3}{|c|}{ Эксперимент } \\
\hline & & & \multirow{2}{*}{$\begin{array}{c}\text { B3LYP } \\
{[17]} \\
v, \mathrm{~cm}^{-1}\end{array}$} & \multirow{2}{*}{$\begin{array}{c}\text { Амплитуда } \\
(I R) \\
\text { (arb. units) } \\
{[17]}\end{array}$} & \multirow{2}{*}{$\begin{array}{c}\text { B3LYP } \\
{[41]} \\
v, \mathrm{~cm}^{-1}\end{array}$} & \multirow{2}{*}{$\begin{array}{l}I R[36] \\
v, \mathrm{~cm}^{-1}\end{array}$} & \multicolumn{2}{|c|}{$\begin{array}{c}\text { Наши данные } \\
v, \mathrm{~cm}^{-1}\end{array}$} \\
\hline & & & & & & & $I R$ & $R$ \\
\hline$v_{2}\left(\mathrm{PO}_{4}\right)$ & $\begin{array}{l}I R, R \\
I R, R \\
I R, R \\
I R, R \\
\quad R \\
\quad R\end{array}$ & $\begin{array}{c}A \\
A \\
E_{1} \\
E_{1} \\
E_{2} \\
E_{2}\end{array}$ & $\begin{array}{l}459 \\
489 \\
437 \\
475 \\
440 \\
457\end{array}$ & $\begin{array}{c}0.2 \\
40.4 \\
2.4 \\
34.6 \\
0 \\
0\end{array}$ & $\begin{array}{l}506 \\
527 \\
476 \\
515 \\
482 \\
515\end{array}$ & 462 & $\begin{array}{c}542 \\
-(473)\end{array}$ & $\begin{array}{l}454 \\
432\end{array}$ \\
\hline$v_{4}\left(\mathrm{PO}_{4}\right)$ & $\begin{array}{c}I R, R \\
I R, R \\
I R, R \\
I R, R \\
I R, R \\
I R, R \\
R \\
R \\
R\end{array}$ & $\begin{array}{c}A \\
A \\
A \\
E_{1} \\
E_{1} \\
E_{1} \\
E_{2} \\
E_{2} \\
E_{2}\end{array}$ & $\begin{array}{l}566 \\
599 \\
621 \\
573 \\
600 \\
609 \\
566 \\
588 \\
633\end{array}$ & $\begin{array}{c}1095.5 \\
0.1 \\
4.2 \\
691.2 \\
166.7 \\
735.2 \\
0 \\
0 \\
0\end{array}$ & $\begin{array}{l}599 \\
630 \\
656 \\
602 \\
632 \\
639 \\
591 \\
622 \\
663\end{array}$ & $\begin{array}{c}566 \\
660-520 \\
\\
632 \\
602\end{array}$ & $\begin{array}{c}566 \\
(558) \\
586 \\
(577) \\
612 \\
601\end{array}$ & $\begin{array}{l}582 \\
608 \\
617\end{array}$ \\
\hline$v_{1}\left(\mathrm{PO}_{4}\right)$ & $\begin{array}{l}I R, R \\
I R, R \\
\quad R\end{array}$ & $\begin{array}{c}A \\
E_{1} \\
E_{2}\end{array}$ & $\begin{array}{l}972 \\
972 \\
974\end{array}$ & $\begin{array}{c}2.62 \\
360.2 \\
0\end{array}$ & $\begin{array}{l}991 \\
990 \\
993\end{array}$ & 962 & $966(-)$ & 965.4 \\
\hline$v_{3}\left(\mathrm{PO}_{4}\right)$ & $\begin{array}{c}I R, R \\
I R, R \\
I R, R \\
I R, R \\
I R, R \\
I R, R \\
\quad R \\
R \\
R\end{array}$ & $\begin{array}{l}A \\
A \\
A \\
E_{1} \\
E_{1} \\
E_{1} \\
E_{2} \\
E_{2} \\
E_{2}\end{array}$ & $\begin{array}{l}1054 \\
1064 \\
1096 \\
1045 \\
1068 \\
1110 \\
1041 \\
1077 \\
1081\end{array}$ & $\begin{array}{c}4686.4 \\
136.6 \\
1.0 \\
8207.8 \\
97.6 \\
755.8 \\
0 \\
0 \\
0\end{array}$ & $\begin{array}{l}1088 \\
1111 \\
1127 \\
1080 \\
1107 \\
1146 \\
1081 \\
1109 \\
1126\end{array}$ & $\begin{array}{c}1190-976 \\
1042 \\
1091\end{array}$ & $\begin{array}{c}1032(-) \\
(1093) \\
1064 \\
(1042) \\
(1075) \\
1106(1118)\end{array}$ & $\begin{array}{c}1034 \\
1042.6 \\
1060.3 \\
1053.4 \\
1081.6 \\
\\
1090 \\
1104 \\
1112\end{array}$ \\
\hline
\end{tabular}

Примечание. Обозначения: IR (ИК), $R$ (Раман-КР). Частота в скобках (v) дана для вектора $\mathbf{E} \| \mathbf{c}$, без скобок - для $\mathbf{E} \perp \mathbf{c}$. Черта $(-)$ означает отсутствие полосы в спектре $\mathbf{E} \| \mathbf{c}$, черта без скобок - для $\mathbf{E} \perp \mathbf{c}$.

отмеченное выше, указывает на слабую зависимость симметрии тетраэдра $\mathrm{PO}_{4}$ от замещения иона фтора на ион хлора (табл. 1).

При рассмотрении рис. 7 обращает на себя внимание смещение (относительно спектров 1,2) наиболее интенсивной полосы $v_{3}\left(\mathrm{PO}_{4}\right)$ в низкочастотную область и ее уширение для спектров, полученных с помощью техники $\mathrm{KBr}$, тогда как соответствующие параметры менее интенсивной полосы $v_{2}$ меняются менее значительно. Частоты еще более слабых полос $v_{4}$ и $v_{1}$ для сопоставляемых образцов практически не изменяются. На формирование контура полосы $v_{3}$ при использовании техники $\mathrm{KBr}$ влияет ряд факторов: коэффициент поглощения, коэффициент рассеяния и параметр, учитывающий влияние внутреннего поля световой волны на поглощательную способность вещества [34]. Второй и третий факторы определяются величиной показате- ля преломления, который в окрестности полосы $v_{3}$ меняется в широких пределах $n=0.14-7.0$, причем эти оба фактора приводят в спектрах, полученных с помощью техники $\mathrm{KBr}$, к смещению центра полосы $v_{3}$ (спектры 3-4, рис. 7) в низкочастотную область. Это указывает на важную роль аномальной дисперсии, которая вносит свой дополнительный вклад в формирование контура интенсивных полос поглощения при исследовании порошков с помощью техники $\mathrm{KBr}$. $\mathrm{B}$ качестве альтернативы технике $\mathrm{KBr}$ для исследования порошков часто используются методы диффузного отражения [35] и фотоакустической спектроскопии [36], однако аномальная дисперсия в обоих случаях определенным образом [33,35,37] реагирует на измеряемую этими методами абсорбционную способность вещества в окрестности интенсивных полос поглощения. В ИК спектре (рис. 7) образца S2 (табл. 1) можно обнаружить 
слабую полосу $745 \mathrm{~cm}^{-1}$, что указывает на присутствие гидроксильной группы $[38,39]$. Группа ОН . . . F присутствует в спектре КР образца S2 и имеет полосу валентных колебаний $\sim 3540 \mathrm{~cm}^{-1}$ [38]. Полоса $745 \mathrm{~cm}^{-1}$ либрационных колебаний, относящаяся к группе $\mathrm{OH}$, также присутствует в спектрах абсорбции образцов S5 и S9, которые исследовались в виде порошков [28]. Полоса $745 \mathrm{~cm}^{-1}$ обладает, как видно из поляризационных спектров $k_{o}$ и $k_{e}$ (рис. 7, вставка в центре), сильным дихроизмом. Ее интенсивность у образца S2 наибольшая для вектора $\mathbf{E} \| \mathbf{c}$ (рис. 7), тогда как для вектора $\mathbf{E} \| \mathbf{c}$ интенсивность полосы $745 \mathrm{~cm}^{-1}$ равна нулю. Следовательно, дипольный момент перехода этого колебания ориентирован перпендикулярно оси с кристалла, тогда как ион $\mathrm{OH}^{-}$расположен параллельно оси c $[10,11]$. В спектре образца S8 присутствуют полоса $866 \mathrm{~cm}^{-1}$ (рис. 7) и дублет 1424-1454 $\mathrm{cm}^{-1}$, которые относятся к колебаниям $v_{2}\left(\mathrm{CO}_{3}\right)$ и $v_{3}\left(\mathrm{CO}_{3}\right)_{3}$. Присутствие группы $\mathrm{CO}_{3}$ в спектре образца $\mathrm{S} 8$ подтверждается данными анализа химического состава этого образца (табл. 1).

Максимумы основных частот аниона $\mathrm{PO}_{4}^{3-}$, относящихся к колебаниям $v_{2}\left(\mathrm{PO}_{4}\right), v_{4}\left(\mathrm{PO}_{4}\right), v_{1}\left(\mathrm{PO}_{4}\right)$ и $v_{3}\left(\mathrm{PO}_{4}\right)$ тетраэдра $\mathrm{PO}_{4}$ в поляризационных спектрах $k_{o}(v)$ и $k_{e}(v)$ (табл. 4, рис. 5-7), были интерпретированы в соответствии с данными расчета частот, интенсивностей [17], а также дихроизма полос, измеренного в нашем эксперименте. Из рис. 7 видно, что полосы 965 и $473 \mathrm{~cm}^{-1}$, отвечающие симметричному колебанию $v_{1}\left(\mathrm{PO}_{4}\right)$ и деформационному $v_{2}\left(\mathrm{PO}_{4}\right)$ соответственно, сильно дихроичны. Дипольные моменты перехода колебания $v_{1}$ и $v_{2}$ (исходя из симметрии тетраэдра $\mathrm{PO}_{4}$ ) ориентированы перпендикулярно и параллельно оси с кристалла соответственно. Следовательно, ось симметрии тетраэдра $\mathrm{PO}_{4}$, проходящая через его вершину (атом Р) направлена перпендикулярно к оси с. Моду $v_{1}\left(\mathrm{PO}_{4}\right)$ следует отнести к параллельному колебанию, поскольку дипольный момент этой моды в процессе колебания изменяется параллельно оси симметрии тетраэдра $\mathrm{PO}_{4}[40]$.

Полоса антисимметричного колебания $v_{3}\left(\mathrm{PO}_{4}\right)$ в ИК спектре образца S2 расщепляется на две основные компоненты (рис. 7) с максимумами $k_{e 1032 \mathrm{~cm}^{-1}}$ и $k_{o 1042 \mathrm{~cm}^{-1}}$. Мода с частотой $1032 \mathrm{~cm}^{-1}$ может быть отнесена к параллельному колебанию и с учетом ее сильного дихроизма по симметрии принадлежит к $A$-типу. Моду с частотой $1042 \mathrm{~cm}^{-1}$ с учетом ее слабо выраженного дихроизма следует отнести к перпендикулярному колебанию $E_{1}$-типа. При сравнении эксперимента с расчетом (рис. 8) заметно, что полоса $v_{3}$, вычисленная для неориентированного образца S2 из поляризационных ИК спектров $k_{o}(v)$ и $k_{e}(v)$, несимметрична и заметно шире, чем полученная расчетом методом $a b$ initio в рамках модели B3LYP $[17,41]$. Причем при нормировке основной полосы $1042 \mathrm{~cm}^{-1}$ для спектров рис. $8, a, b$ интенсивность колебания $v_{3}$ с частотой $\sim 1093 \mathrm{~cm}^{-1}$ существенно больше для образца S2 по сравнению с
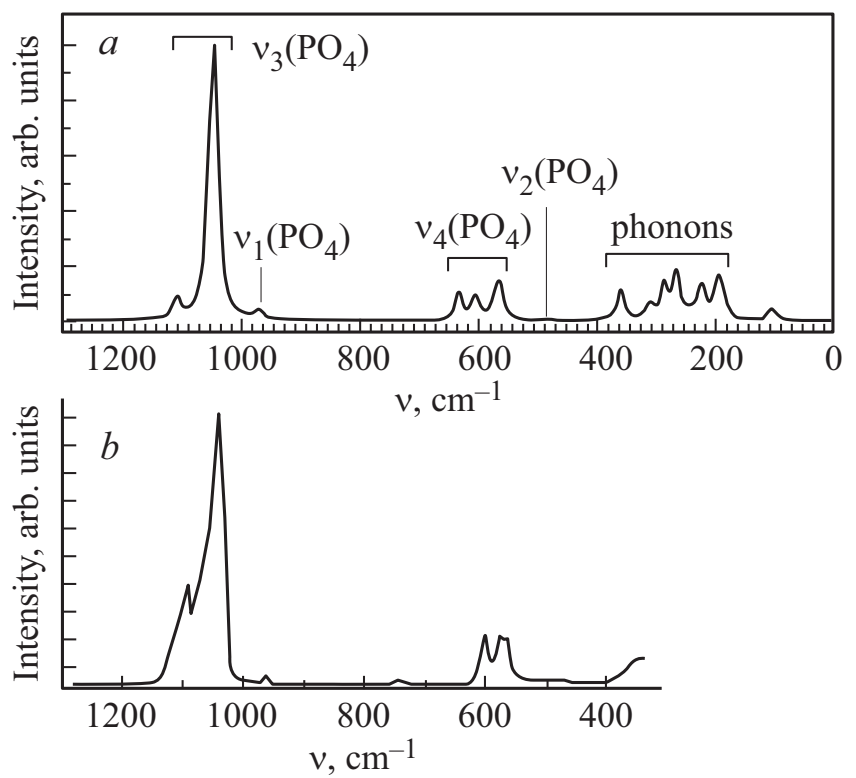

Рис. 8. Спектры абсорбции апатита в ИК области: $a-$ расчет, метод $a b$ initio [17], $b$ - эксперимент, спектр представлен для неориентированного образца S2 (табл. 1), вычислен на основе значений $k$ для двух ориентаций вектора $\mathbf{E} \| \mathbf{c}$ и $\mathbf{E} \perp \mathbf{c}$.

расчетом. Исходя из этого наблюдения можно предположить, что электронная плотность на определенном атоме кислорода тетраэдра $\mathrm{PO}_{4}$, задаваемая моделью B3LYP, несколько занижена, что приводит к уменьшению ионности связи $\mathrm{P}-\mathrm{O}$ и, как следствие, к уменьшению ее дипольного момента перехода. Большая полуширина полосы $v_{3}$ в спектре образца $\mathrm{S} 2$ (рис. $8, b$ ) по сравнению с расчетной кривой (рис. 8, $a$ ) связана с расщеплением колебательных мод $v_{3}$, которые ориентированы вдоль и поперек оси с. Дополнительным фактором уширения полосы $v_{3}$ является взаимодействие колебательных мод тетраэдров $\mathrm{PO}_{4}$ с кристаллической решеткой природного кристалла FAp. На основании анализа полуширины полос колебательных мод $v_{1}, v_{2}$ и $v_{3}$ (рис. 7) можно предположить, что такое взаимодействие в большей мере затрагивает перпендикулярные колебания, имеющие симметрию $E$, чем параллельные с симметрией $A$. Для колебательных мод с симметрией $E$ дипольный момент перехода в отличие от мод $A$ имеет две составляющие вектора дипольного момента, направленные вдоль и поперек оси симметрии тетраэдра $\mathrm{PO}_{4}$, что увеличивает вероятность колебательного взаимодействия этого типа колебаний с кристаллической решеткой кристалла.

В целом можно отметить, что частоты в поляризационных спектрах монокристалла FAр (образец S2), полученные из спектров ИК отражения с помощью метода КК, вполне хорошо согласуются с расчетом [17,39], в меньшей мере это можно отнести к интенсивностям полос, если сопоставлять относительные интенсивности полос, полученных в эксперименте, с расчетом. 
Таким образом, для лучшего согласия теории с экспериментом теоретические методы расчета должны позволять учитывать зависимость колебательных мод тетраэдров $\mathrm{PO}_{4}$ от анизотропии апатита и взаимодействие этих мод с кристаллической решеткой апатитов.

Образец S2 получен из ГЕОХИ РАН при содействии д.ф.-м.н. Г.В. Юхневича, образец S1 предоставлен Иркутским государственным университетом, образцы S3, S4 получены из коллекции проф. Р.С. Ханукоева. Исследования спектров КР проведены с использованием оборудования ресурсного центра Научного парка СПбГУ „Оптические и лазерные методы исследования вещества“. Приношу благодарность специалисту по рамановской спектроскопии Д.В. Панькину за измерения спектров КР.

Работа выполнена при финансовой поддержке Министерства образования и науки Российской Федерации (проект 16.1651.2017/4.6).

\section{Список литературы}

[1] Steinbruegge K.B., Henningsen T., Hopkins R.H., Mazelsky R., Melamed N.T., Riedel E.P., Roland G.W. // Appl. Optics. 1972. V. 11. P. 999-1012.

[2] Qi S., Huang Y., Tsuboi T., Huang W., Seo H.J. // Opt. Mater. Express. 2014. V. 4. P. 397-402.

[3] Ткаченко Н.В., Ольховик Л.П., Камзин А.С. // ФТТ. 2011. T. 53. № 8. C. 1512-1517.

[4] Thakre P.S., Gedam S.C., Dhobl S.J., Atram R.G. // J. Luminesc. 2011. V. 131. P. 2683-2689.

[5] Hughes J.M., Rakova J. // Phosphates: Geochemical, Geobiological and Material Importance. Reviews in Mineralogy and Geochemistry. V. 48. / Ed. by Kohn M.J., Rakovan J., Hughes J.M. Washington, DC: Mineralogical Society of America, 2002. P. 1-12.

[6] Wopenka B.T., Pasteris J.D. // Mater. Sci. and Engin. 2005. V. 25. P. 131. doi: $10.1016 /$ j.msec.2005.01.008

[7] Jiangling Li. Structural Characterization of a Patite-like Materials. A thesis submitted to the School of Metallurgy and Materials for the degree of MRes in Biomaterials. UK, University of Birmingham, 2009.

[8] Плюснина И.И. Инфракрасные спектры минералов. М.: Изд-во Моск. ун-та. 1976. 175 с.

[9] Adler H. // American Mineralogist. 1964. V. 49. P. 1002-1015.

[10] Кнубовец Р.Г., Кисловский Л.Д. // Физика апатита (Исследование апатита методами спектроскопии) / Под ред. Соболева В.С. Новосибирск: Наука, 1975. С. 63.

[11] Veiderma M., Knubovets R., Tonsuaadu K. // Bulletin of the Geological Society of Finland. 2007. V. 70. Parts 1-2. P. 69-75.

[12] Infrared Spectroscopy - Materials Science, Engineering, and Technology / Ed. by Theophanides Theophile. Publisher InTech, 2012. $510 \mathrm{p}$.

[13] Yamagishi K., Onuma K., Suzuki T., Okada F., Tagami J., Otsuki M., Senawangse P. // Nature. 2005. V. 433. P. 819.

[14] Dorozhkin S. // Materials. 2009. V. 2. P. 1975-2045. doi: $10.3390 / \mathrm{ma} 2041975$
[15] Xiaofeng Pang, Hongjuan Zeng, Jialie Liu, Shicheng Wei, Yufeng Zheng // Mater. Sci. and Appl. 2010. V. 1. P. 81-90. doi: $10.4236 / \mathrm{msa} .2010 .12015$

[16] Schuetz R., Fix D., Schade U., Aziz E.F., Timofeeva N., Weinkamer R., Masic A. // Molecules. 2015. V. 20. P. 5835 5850. doi: 10.3390/molecules20045835

[17] Ulian G., Valgre G., Corno M., Ugliengo P. // American Mineralogist. 2017 (in press). dx.doi.org/10.2138/am.2013.4315

[18] Ulian G. Dottorato di ricerca in Scienzedella Terra „Ab Initio Quantum Mechanical Investigation of Structural and Chemical-physical Properties of Selected Minerals for Minero-petrological, Structural Ceramic and Biomaterial Applications“. Universita di Bologna, 2014.

[19] Beery E., Fitzgerald A.J., Zinov'ev N.N. et al. // Proceed. SPIE: Medical Imaging 2003: Physics of Medical Imaging. 2003. V. 5030. P. 459-470. doi.org/10.1117/12.479993

[20] Howell B.F., JR., Licastro P.E. // American Mineralogist. 1961. V. 46. P. 269-288

[21] Kravitz L.C., Kingsley J.D., Elkin E.L. // J. Chem. Phys. 1968. V. 19. P. 4600.

[22] Zuerlein M.J., Fried D, Featherstone J.D.B., Seka W. // IEEE J. Selected Topics in Quantum Electronics. 1999. V. 5. № 4. P. 1083-1089.

[23] Duplain G., Boulay R., Belanger P.A. // Appl. Opt. 1987. V. 26. № 20. P. 4447-4451. doi: 10.1364/AO.26.004447

[24] Clark R.N., Swayze G.A., Wise R., Livo K.E., Hoefen T.M., Kokaly R.F., Sutley S.J. // USGS Digital Spectral Library splib06a. U.S. Geological Survey. Data Series 231. 2007. Электронный ресурс. Режим доступа: https://crustal.usgs.gov/speclab

[25] Ostrooumov M., Lasnier B., Lefrant S. Database: Infrared Reflection Spectrometry of Minerals and Gems. Catalogue of the Spectrum Nantes 1993-2009. Электронный ресурс. Режим доступа: http://www.mineralog.net/?page_id=10

[26] De Mul F.F.M., Hottenhuis M.H.J., Bouter P., Greve J., Arends J., Bosch J.J.T. // J. Dent. Res. 1986. V. 65. P. 437-440.

[27] De Aza P.N., Santos C., Pazo A., De Aza S., Cusco R., Artus L. // Chem. Materials. 1997. V. 9. P. 912-915.

[28] RRUFF Database Raman, X-ray, Infrared, and Chemistry Files. Электронный ресурс. Режим доступа: http://www.rruff.info

[29] Альперович Л.И. Метод дисперсионных соотношений и его применение для определения оптических характеристик. Душанбе: Ирфон, 1973.

[30] Kitamura R., Pilon L., Jonasz M. // Appl. Optics. 2007. V. 46. № 33. P. $8118-8133$.

[31] Smith D.Y. Dispersion Theory, Sum Rules, and Their Application to the Analysis of Optical Data // Handbook of Optical Constants of Solids. V. 1 / Ed. by Palik E.D. San Diego: Acad. Press, 1985. P. 35.

[32] Золотарев В.М., Морозов В.Н., Смирнова Е.В. Оптические постоянные природных и технических сред. Л.: Химия, 1984. $218 \mathrm{c}$.

[33] Золотарев В.М. // Опт. и спектр. 2017. Т. 122. № 5. С. 772 779. 
[34] Поль Р.В. Оптика и атомная физика. М.: Наука, 1966. 552 с.

[35] Dahm D.J., Dahm K.D. Interpreting Diffuse Reflectance and Transmittance: A Theoretical Introduction to Absorption Spectroscopy of Scattering Materials. Chichester, UK: NIR Publications, 2007.

[36] Rehman I., Bonfield W. // J. Materials Sciences-Materials in Medicine. 1997. № 8(1). P. 1-4.

[37] Борен К., Хафен Д. Поглощение и рассеяние света малыми частицами. М.: Мир, 1986. 660 с.

[38] Золотарев В.М. // Опт. и спектр. 2017. Т. 123. № 1. С. $37-$ 47.

[39] Freund F., Knobel R.M. // J. Chem. Soc., Dalton Transactions. 1977. V. 11. P. 1136-1140.

[40] Бенуэлл К. Основы молекулярной спектроскопии. М.: Мир, 1985.

[41] Corno M., Busco C., Civalleri B., Ugliengo P. // Phys. Chem. Chem. Phys. 2006. V. 8. P. 2464-2472. 